\title{
Blood culture-negative endocarditis caused by Bartonella henselae: a case report
}

\author{
Tsukasa Ohno $^{1} \cdot$ Shunei Saito $^{1}$ (i) $\cdot$ Eri Iwata $^{2} \cdot$ Sen Ushida ${ }^{2} \cdot$ Ryohei Otsuka $^{1} \cdot$ Ken Miyahara $^{1} \cdot$ Akio Matsuura $^{1}$
}

Received: 18 June 2021 / Revised: 31 August 2021 / Accepted: 1 September 2021 / Published online: 5 November 2021

(c) Indian Association of Cardiovascular-Thoracic Surgeons 2021

\begin{abstract}
Bartonella henselae is well known as a causative organism of cat scratch disease. Although this bacterium infrequently involves the heart, the diagnosis is difficult to confirm. A 75-year-old woman who had a pet cat presented with pancytopenia, hepatosplenomegaly, and low-grade fever. Echocardiography depicted sessile nodules on the aortic valve. C-reactive protein concentration was low, and leukocytosis was not seen. Two sets of blood culture turned out negative. However, elevated $B$. henselae immunoglobulin $\mathrm{G}$ titer led us to the diagnosis of infective endocarditis. Minocycline was administered orally in combination with intravenous administration of gentamicin as an antimicrobial treatment. The patient underwent aortic valve replacement 2 months after her initial visit. Warthin-Starry silver staining did not show any bacterial bodies. The culture of the vegetation tissue was negative. Polymerase chain reaction testing of the excised valve tissue detected the deoxyribonucleic acid of the organism. The postoperative course was uneventful, and the patient was discharged home.
\end{abstract}

Keywords Bartonella henselae $\cdot$ Endocarditis $\cdot$ Aortic valve $\cdot$ Serology $\cdot$ Polymerase chain reaction

\section{Introduction}

Bartonella henselae is well known as a causative organism of cat scratch disease (CSD). Although this bacterium infrequently becomes the etiologic agent of infective endocarditis, it is difficult to confirm the diagnosis. Herein, we describe a case of blood culture-negative endocarditis (BCNE) caused by $B$. henselae.

\section{Case report}

A 75-year-old woman was referred to the division of hematology in our institution because of pancytopenia, general fatigue, low-grade fever, and lower leg edema (day 0 ). The patient had a history of diabetes mellitus as well as hypertension and was keeping a cat, which used to be a stray.

Tsukasa Ohno

t-ono@med.nagoya-u.ac.jp

1 Division of Cardiovascular Surgery, Ichinomiya Municipal Hospital, 2-2-22 Bunkyo, Ichinomiya, Aichi 491-8558, Japan

2 Division of Hematology, Ichinomiya Municipal Hospital, Ichinomiya, Japan
She had not taken any antibiotics before presentation to our institution. Blood analysis at the initial visit was as follows: white blood cell, $4600 / \mu \mathrm{L}$ (segmented cell, 70\%); hemoglobin, $8.2 \mathrm{~g} / \mathrm{dL}$; platelet, $70.0 \times 10^{3} / \mu \mathrm{L}$; aspartate aminotransferase, $21 \mathrm{IU} / \mathrm{L}$; alanine transaminase, $9 \mathrm{IU} / \mathrm{L}$; creatinine, $0.71 \mathrm{mg} / \mathrm{dL}$; blood urea nitrogen, $20.2 \mathrm{mg} / \mathrm{dL}$; C-reactive protein (CRP), $3.46 \mathrm{mg} / \mathrm{dL}$; immunoglobulin (Ig) G, $1975 \mathrm{mg} / \mathrm{dL} ; \mathrm{IgA}, 90 \mathrm{mg} / \mathrm{dL}$; and IgM, $29 \mathrm{mg} / \mathrm{dL}$. The urine test was positive for protein. On auscultation, slight systolic murmur was audible. Transthoracic echocardiogram revealed sessile nodules on the right coronary cusp $(18 \times 8 \mathrm{~mm})$ as well as on the left coronary cusp $(6 \times 6 \mathrm{~mm})$ (Fig. 1a). The ejection fraction was $55 \%$, and mild to moderate aortic regurgitation was detected. Computed tomography showed moderate hepatosplenomegaly (Fig. 1b). Although bone marrow aspiration was performed for possible lymphocytic leukemia, no atypical cells were observed. The two sets of blood culture submitted on day 0 came out negative. On day 7 , furosemide $(20 \mathrm{mg} /$ day $)$ was started as a treatment for lower leg edema. On day 12, in consideration of BCNE, Bartonella antibody test using immunofluorescence assay (IFA) (Focus Diagnostics, Cypress, CA, USA) was carried out. Minocycline $(200 \mathrm{mg} /$ day $)$ was initiated on the same day as a diagnostic therapy. On day 35 , elevated $B$. henselae $\mathrm{IgG}$ titer (1:1024) was reported. IgM titer was low. On 

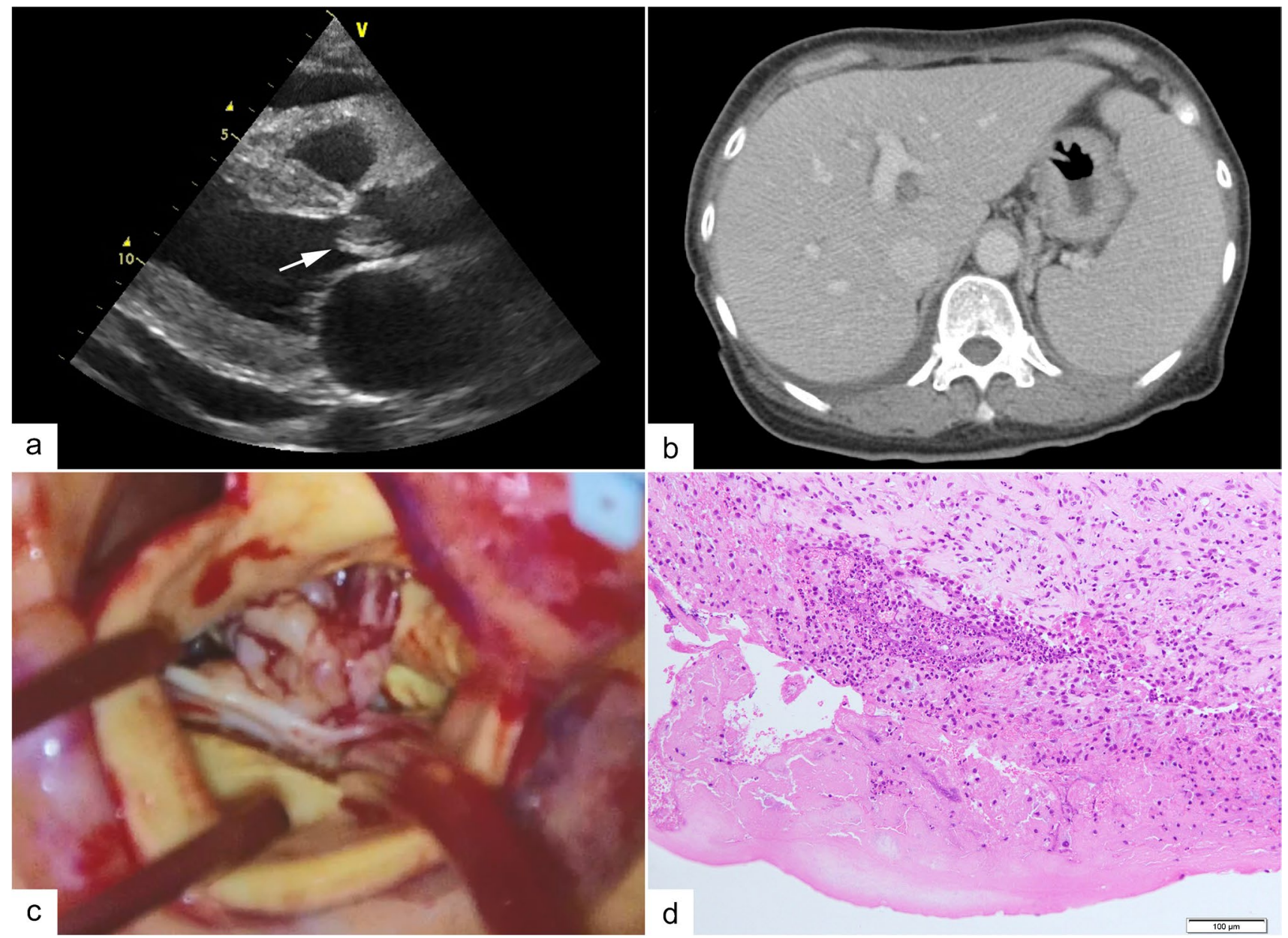

Fig. 1 a Long-axis view of transthoracic echocardiogram. The arrow shows the vegetation on the aortic valve. b Computed tomography. Hepatosplenomegaly is observed. c Intraoperative image. Two fragile vegetations were noted on the ventricular side of the right and left

day 56, the patient complained of dyspnea on exertion. The second echocardiogram revealed no change in size of the vegetation, exacerbation of aortic regurgitation (moderate), reduced ejection fraction $(45 \%)$, and pericardial effusion. The patient was admitted to the cardiovascular surgery ward, and a continuous infusion of intravenous furosemide was started. On day 59, gentamicin was administered intravenously (initially $120 \mathrm{mg} / \mathrm{day}$, later decreased to $60 \mathrm{mg} / \mathrm{day}$ based on therapeutic drug monitoring) in addition to minocycline given orally, considering $B$. henselae as the target organism. Although the blood B. henselae polymerase chain reaction (PCR) test (Genesig PCR Kit by Primerdesign Ltd, Camberley, England) was negative, BCNE caused by the organism was strongly suspected. After taking gradual exacerbation of aortic regurgitation and left ventricular function into consideration, we concluded that the infection had not been controlled despite minocycline administration. Surgery was carried out on day 69 . coronary cusps. d Histopathological image. Infiltration of neutrophils and lymphocytes as well as fibrin precipitation is recognized. Original magnification $\times 200$

Following median sternotomy, extracorporeal circulation was established by ascending aortic return and bicaval drainage. On opening of the aorta, fragile vegetations on the ventricular side of right and left coronary cusps were observed (Fig. 1c). Neither the annulus nor the mitral valve was involved. The leaflets were excised carefully, and a 21-mm Inspiris Resilia bioprosthesis (Edwards Lifescience, Irvine, CA, USA) was implanted in the supra-annular position.

Histopathology of the valve tissue stained with hematoxylin and eosin revealed infiltration of neutrophils and lymphocytes as well as precipitation of fibrin, which were consistent with infective endocarditis (Fig. 1d). Warthin-Starry silver staining did not show any bacterial bodies. The culture of the vegetation tissue was negative. However, PCR analysis of the vegetation was positive, detecting deoxyribonucleic acid (DNA) of $B$. henselae.

On day 75, gentamicin was discontinued. The postoperative course was uneventful, and the patient was discharged 
on day 87. Blood test results were as follows: white blood cell, $3700 / \mu \mathrm{L}$; hemoglobin, $11.1 \mathrm{~g} / \mathrm{dL}$; platelet, $153 \times 103$ $/ \mu \mathrm{L}$; and CRP, $0.17 \mathrm{mg} / \mathrm{dL}$. On day 96 , minocycline was stopped to complete the treatment.

\section{Discussion}

B. henselae, formerly Rochalimaea, is a bacterium that is the causative agent of CSD. Humans usually get infected through scratches or bites of infected cats. Canine to human transmission is anecdotally reported. The disease is typically characterized by regional lymphadenopathy and fever. Although the heart involvement is known, the diagnosis is often difficult to confirm. Bartonella species account for 9-10\% of BCNE [1, 2]. Houpikian et al. [3] reported that among 71 cases of Bartonella endocarditis, B. quintana was responsible for 53 (75\%) cases and B. henselae for 17 (24\%) cases. The clinical presentations in the $B$. henselae group were as follows: fever $(100 \%)$, body weight loss $(40 \%)$, heart failure (53\%), splenomegaly (40\%), hepatomegaly (7\%), arterial embolism (47\%), leukocytosis (38\%), anemia (55\%), thrombocytopenia (33\%), renal failure (50\%), and elevated erythrocyte sedimentation rate (83\%) [3].

$B$. henselae is a Gram-negative rod, which is culturable on chocolate agar or blood agar in $5 \% \mathrm{CO}_{2}$ incubator at $35-37^{\circ} \mathrm{C}$ [4]. However, owing to its fastidious nature, $2-3$ weeks is required for incubation. Thus, the bacterium cannot be easily isolated from clinical specimens. Blood culture methods have a sensitivity as low as $20 \%$ for diagnosing Bartonella endocarditis, while tissue culture of excised valves has a similarly low sensitivity of $30 \%$ [3]. Histopathologically, Warthin-Starry silver staining has frequently been employed and reveals black-stained bacteria in the affected valve tissue. The reported sensitivity in Bartonella endocarditis is $46 \%$ [5]. However, this staining method also detects Helicobacter pylori, Legionella pneumophila, spirochetes, and Klebsiella species, but does not provide a definitive diagnosis.

For the above reasons, serological as well as PCR testing is essential to confirm B. henselae infection. IgM and IgG antibodies are measured by commercially available IFA. In the kit used, the cutoff indicating acutephase infection was as follows: (1) IgM titers $\geq 1: 20$ or IgG titers $\geq 1: 256$, (2) initial specimen $\operatorname{IgG}$ titer $\geq 1: 64$ and $<1: 256$, and (3) second specimen (drawn 10-21 days after) titer $\geq 1: 256$ or a fourfold increase. Fournier et al. [6] reported that IgG titer of $\geq 1: 800$ was highly indicative of current infection in patients with endocarditis. The specificity and sensitivity of serological analysis vary significantly across the literature probably due to crossreactivity and between-kit variability [7]. By contrast, PCR test can identify the DNA of $B$. henselae. According to Edouard et al. [5], among patients with endocarditis caused by $B$. henselae or $B$. quintana, the sensitivity was $33 \%$ and $92 \%$ in blood samples and surgically resected valve specimens, respectively. PCR testing of the valve tissue appears to be most useful in confirming the diagnosis, as was in the presented case. Notably, the modified Duke criteria, which mainly rely on blood culture positivity, show a lower accuracy for early diagnosis of endocarditis caused by Bartonella species.

Bartonella species are considered to evade the host immune system because of their intra-erythrocytic propagation and biofilm formation [8]. Thus, the use of at least two antibiotics has been advocated. The Sanford guide [9] recommended $200 \mathrm{mg} /$ day of doxycycline for 6 weeks combined with $3 \mathrm{mg} / \mathrm{kg} /$ day of gentamicin for 2 weeks in patients with endocarditis. Since doxycycline was unavailable in our institution, minocycline was employed as an alternative. As regards its intractability to antimicrobial treatment, surgical resection of the infected valve tissue should always be considered.

Delayed diagnosis of endocarditis may cause further destruction of the valve, aggravation of heart failure, and annular abscess, which result in poor surgical outcomes. In our case, CRP before starting antimicrobial treatment remained low between 0.94 and $3.46 \mathrm{mg} / \mathrm{dL}$, and no leukocytosis was detected. Ribeyrolles et al. [10] reported that CRP at admission was $<2 \mathrm{mg} / \mathrm{dL}$ in 13 (2.8\%) of 469 patients with endocarditis. Not all patients with endocarditis present with elevated CRP or leukocytosis. Moreover, especially in patients with BCNE, the use of transesophageal echocardiography may help identify the progression of valve destruction and aortic regurgitation.

In conclusion, when an echocardiogram shows a mass on the valve leaflet, whereas blood culture is negative, serological as well as PCR testing should be carried out to screen for infection caused by fastidious bacteria, including Bartonella species.

Author contribution All authors contributed to the diagnosis or the treatment of the disease. The first draft of the manuscript was written by Tsukasa Ohno and was critically revised by Shunei Saito. All authors commented on previous versions of the manuscript. All authors read and approved the final manuscript.

Funding None.

Data availability Not applicable.

Code availability Not applicable.

\section{Declarations}

Ethics approval Ethical approval was waived by the local Ethics Committee of Ichinomiya Municipal Hospital in view of the retrospective 
nature of the study and all the procedures being performed were part of the routine care.

Informed consent Written informed consent was obtained from the patient for publication of this case report and accompanying images.

Conflict of interest The authors declare no competing interests.

\section{References}

1. Brouqui P, Raoult D. Endocarditis due to rare and fastidious bacteria. Clin Microbiol Rev. 2001;14:177-207.

2. Lamas CC, Eykyn SJ. Blood culture negative endocarditis: analysis of 63 cases presenting over 25 years. Heart. 2003;89:258-62.

3. Houpikian P, Raoult D. Blood culture-negative endocarditis in a reference center: etiologic diagnosis of 348 cases. Medicine. 2005;84:162-73.

4. Koehler JE, Quinn FD, Berger TG, LeBoit PE, Tappero JW. Isolation of rochalimaea species from cutaneous and osseous lesions of bacillary angiomatosis. N Engl J Med. 1992;327:1625-31.

5. Edouard S, Nabet C, Lepidi H, Fournier P-E, Raoult D. Bartonella, a common cause of endocarditis: a report on 106 cases and review. J Clin Microbiol. 2015;53:824-9.
6. Fournier P-E, Mainardi J-L, Raoult D. Value of microimmunofluorescence for diagnosis and follow-up of bartonella endocarditis. Clin Diagn Lab Immunol. 2002;9:795-801.

7. Allizond V, Costa C, Sidoti F, et al. Serological and molecular detection of bartonella henselae in specimens from patients with suspected cat scratch disease in Italy: a comparative study. PloS One. 2019;14:e211945.

8. Okaro U, Addisu A, Casanas B, Anderson B. Bartonella species, an emerging cause of blood-culture-negative endocarditis. Clin Microbiol Rev. 2017;30:709-46.

9. Gilbert DN, Chambers HF, Eliopoulos GM, Saag MS, Pavia AT. The Sanford guide to antimicrobial therapy 2020. Sperryville: Antimicrobial Therapy; 2020.

10. Ribeyrolles S, Ternacle J, San S, et al. Infective endocarditis without biological inflammatory syndrome: description of a particular entity. Arch Cardiovasc Dis. 2019;112:381-9.

Publisher's note Springer Nature remains neutral with regard to jurisdictional claims in published maps and institutional affiliations. 